\title{
PERSPECTIVA ANTROPOLÓGICA DE UN CASO DE ORTOREXIA NERVIOSA
}

\author{
Eva Molina Alén \\ Enfermera, Licenciada en Antropología Social y Cultural \\ Profesora Asociada de la Universidad Rey Juan Carlos (URIC) \\ Supervisora de la UHB de Psiquiatría del Hospital de Mostoles.
}

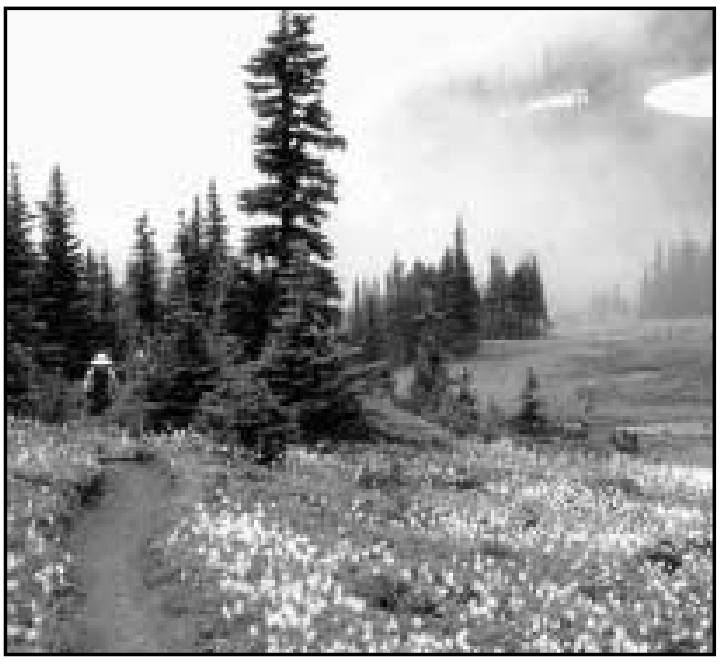

\section{ANTHROPOLOGICAL PERSPECTIVE OF A CASE OF ORTHOREXIA NERVOSA}

\section{SUMMARY}

$\mathrm{I}$ $\mathrm{n}$ this work, the anthropologic perspective has allowed us a deep understanding and interpretation of this case, without which the sociocultural factors that have affected the phenomenology of the case would NOT HAVE BEEN CONSIDERED OR would HAVE BEEN OVERLOOKED.

A new eating disorder is emerging, the socalled "Orthorexia Nervosa(ON)". Orthorexia is related to the extreme worry in the search for health concentrated in eating as healthily and purely as possible. It consists of an excessive obsession and worry for healthy, natural or biological intake, which leads the individual to exclusively consume food coming from ecological agriculture, free from transgenic or artificial components, pesticides or herbicides.

In the developed societies, where the problem is not the lack of food but the excess, eating disorders are becoming more common. $\mathrm{ON}$ seems not to affect the marginal sectors but rather on the contrary since this type of food is much more expensive than the usual one and also more difficult to obtain.

KEY WORDS: Orthorexia nervosa; vegetarianism; Religion; Hinduism; Yoga; Humankind evolution.

\section{RESUMEN}

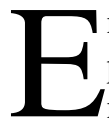
n este trabajo, la mirada antropológica ha permitido realizar las profundizaciones necesarias proporcionando comprensión e interpretación al caso clínico que nos ocupa, sin la cual no se contemplarían o se pasarían por alto los factores socioculturales que han incidido en la fenomenología del caso.

Un nuevo trastorno de la conducta alimentaria (TCA) está emergiendo, es el denominado "Ortorexia nerviosa (ON)". La ortorexia está relacionada con la preocupación extrema o búsqueda de la salud centrada en comer lo más sano posible. Consiste en una obsesión y preocupación desmedidas por lo sano, lo natural o lo biológico, que lleva al individuo a consumir exclusivamente alimentos procedentes de la agricultura ecológica, libres de componentes transgénicos, artificiales, pesticidas o herbicidas.

En las sociedades desarrolladas, en las que el problema no es la falta de alimentos sino el exceso, cada vez inciden más los TCA. La ON parece no afectar a los sectores marginales sino más bien al contrario ya que este tipo de comida es mucho más cara que la normal y más difícil de conseguir.

PALABRAS CLAVES: Ortorexia nerviosa; Vegetarianismo; Religión; Hinduismo; Yoga; Evolución del hombre. 


\section{INTRODUCCIÓN}

$\mathrm{L}$ o que se presenta a continuación pretende demostrar que los estudios de Antropología ofrecen nuevas formas de comprender e interpretar la Enfermería, y es desde un caso de ortorexia nerviosa que destacaré cómo la mirada antropológica ha permitido realizar las profundizaciones necesarias proporcionando explicación y significado, pues, sin ésta nueva perspectiva no se contemplaría o se pasaría por alto la influencia de los factores socioculturales que han incidido en la fenomenología de este caso.

Ahora bien, ¿qué es la ortorexia nerviosa? La ortorexia nerviosa $(\mathrm{ON})$ es un nuevo trastorno de la conducta alimentaria (TCA) que está emergiendo y está relacionado con la preocupación extrema o búsqueda de la salud centrada en comer lo más sano posible. Consiste en una obsesión y preocupación desmedidas por lo sano, lo natural o lo biológico, que lleva al individuo a consumir exclusivamente alimentos procedentes de la agricultura ecológica, libres de componentes transgénicos, artificiales, pesticidas o herbicidas.

A los TCA se les ha adjudicado un origen multifactorial, destacando la importancia de los factores predisponentes para desarrollarla y especialmente a los denominados culturales, motivo que refuerzan aún más la necesidad de estudiarlos desde las ciencias sociales.

\section{OBJETIVOS}

1. OBJETIVO GENERAL: demostrar la importancia de la antropología como disciplina para complementar la formación enfermera $\mathrm{y}$ poder aplicarla en todos los campos.

2. OBJETIVOS ESPECÍFICOS: Proporcionar comprensión e interpretación al caso clínico presentado e identificar los principales factores ideológicos y religiosos y sus influencias en la génesis de la ortorexia nerviosa

\section{DESARROLLO}

La palabra ortorexia, proviene del griego "ortho" que significa justo, recto, correcto y "orexia", apetencia, apetito, con lo que podría definirse como apetito justo o correcto. El creador del término es Steven Bratman [1] médico estadounidense, experto en las medicinas alternativas, que durante años defendió la teoría de una dieta para alcanzar un estado de salud pleno. Según Bratman, cuando la obsesión por comer sano se lleva al extremo, a lo patológico, llega un punto en que todo gira en torno a la comida, controlar lo que comen, imponerse prohibiciones y programar detalladamente las comidas se convierte en una prioridad para poder sentirse seguros, tranquilos y dueños de cada situación (Bratman \& Knight, 2001).

Algunos autores estiman la prevalencia de la ortorexia nerviosa en España en menos de un $1 \%$ de la población [2] siendo más frecuente en mujeres que en hombres, presentándose con una proporción de diez a uno, sobre todo en personas vegetarianas, macrobióticas, crudistas y frutistas.

Hay una evidencia cada vez mayor de que existe una serie de factores socioculturales y biológicos que interactúan entre sí contribuyendo a la presentación de los trastornos de la alimentación, participando en ellos mecanismos psicológicos menos específicos y una vulnerabilidad de la personalidad.

La restricción de alimentos conduce a la malnutrición que a su vez contribuye a cambios físicos $\mathrm{y}$ emocionales que se ponen de manifiesto en forma de alteraciones morfológicas, psíquicas y funcionales tales como la pérdida de peso y la detención del crecimiento entre las morfológicas, la tristeza y el mal humor en las psíquicas, y desequilibrios metabólicos, cardiovasculares y pérdida de capacidad inmunitaria permitiendo la aparición de infecciones oportunistas en las alteraciones funcionales. Será necesario considerar los criterios de hospitalización para los casos de desnutrición severa y valorar las complicaciones como las infecciones, los desequilibrios metabólicos y las alteraciones cardiovasculares (bradicardia y arritmias graves) para seleccionar en qué unidades deben darse los cuidados, en unidades médicas, psiquiátricas o como en algunos casos en las unidades de cuidados intensivos (Fernández, F y Turón, V., 1998).

\section{Caso clínico:}

Se trata de una mujer de 28 años, crudivegana estricta, que acude al hospital debido a su estado crítico de desnutrición. Previo al ingreso, realiza un viaje a la India con una amiga, regresando a 
España con diarreas y mal estado general que requirió ingreso durante unos días en la Unidad de Cuidados Intensivos (IMC: 10.7), luego en el Servicio de Medicina Interna y posteriormente en la Unidad de Psiquiatría. La peculiaridad del caso, radicaba que por un lado, que a paciente sólo aceptaba alimentarse con un tipo muy especial de alimentos y por otro, la importancia de la ideología y creencias que la paciente proyectaba con una gran carga de espiritualidad.

A los 15 años comienza con la restricción de grasas tras consultar con un médico naturista, progresando hasta los 28 años por situaciones de vegetarianismo que van siendo cada vez más radicales hasta convertirse en una vegana crúdivora y frugívora. Se inicia la pérdida paulatina de peso coincidiendo con un aumento en la actividad física relacionado con trabajo de labores en la agricultura ecológica.

Este caso provocó mucho desconcierto en todo el equipo terapéutico pues no cumplía con el canon habitual de la anorexia ni el de la bulimia nerviosa, obligando a todos a realizar una documentación y un trabajo multidisciplinar debido a las características novedosas y desconocidas del caso. Revisada su biografía, cursó estudios universitarios con notas de sobresalientes, ampliando su currículum de postgrado con cursos de nutrición y alimentación, idioma y formación en Yoga, disciplina que impartió como profesora durante muchos años. Durante su estancia hospitalaria, sobresalen sus grandes conocimientos de dietética y de nutrición, acosando científicamente al nutricionista, que se ve acorralado frecuentemente, corroborando su preparación en la disciplina (Catalina, ML., Bote, B., García, F. \& Ríos, B., 2005). La paciente fuerza la negociación alimentaria hasta límites extremos, realizando cambios de opinión frecuentes y manteniendo una actitud oposicionista, que motivan el desbordamiento del equipo de Nutrición y Dietética. Intenta pactar todo lo que come, los alimentos, las mezclas y las cantidades. La dieta pactada está compuesta por: frutos secos, fruta natural, lechuga, pan de cereales y Tofú (queso de soja.). Llama la atención como la afectada, con unos conocimientos tan amplios sobre nutrición y dietética fue capaz de renunciar a un comportamiento alimentario, negando en todo momento su situación grave de desnutrición, ocultando los síntomas de inanición e incluso rechazando la antibioterapia prescrita por la aparición de infecciones oportunistas [3].

Tras una semana de ingreso, el equipo de enfermería informa de una actitud colaboradora, tomando la alimentación pautada por el Servicio de Nutrición, aceptando los aportes minerales y vitamínicos pero rechazando la medicación y el aporte nutritivo adicional. Así mismo, tras un seguimiento, Enfermería, manifiesta que no observa jugueteos con las comidas, ni objetiva vómitos, tomándose adecuadamente los alimentos pactados. La paciente durante las comidas lleva a cabo una serie de ritos, dando a las comidas un aspecto de ceremonia, poniendo las manos sobre los alimentos como bendiciéndolos. Mientras come, medita masticando cada bocado unas 50 veces. Solicita que le aumenten la cantidad de comida, pues en ningún momento se la ha visto contrariada ni rechaza la idea de aumentar de peso.

Los cambios que llaman la atención a su familia coinciden con su iniciación en el Yoga, modificando la forma de vestir, preocupándose por la preparación de la comida, radicalizando sus creencias naturistas, las ideas de contaminación, practicando la meditación y buscando su equilibrio. En todo momento, la paciente argumenta con bastante coherencia sus creencias, defendiendo ser vegetariana como opción, como estilo y forma de vida: "La no violencia se asocia con una dieta natural. Matar animales es malo y no es necesario para el hombre. Lo mejor sería alimentarse de semillas. Las semillas caen de forma natural a la Tierra porque la alimentación crudivegana mejora el mundo, trayendo paz y amor, salud, convivencia y desarrollo personal". Cuesta que la paciente exprese sus emociones, mostrando cierta labilidad cuando se la fuerza a hablar de sus sentimientos o de su vida particular. Siente que no encaja en el mundo pero niega síntomas de tristeza o depresivos: "depresión es una palabra usada bastante a la ligera refiriendo haberse sobrecargado de trabajo, colocándose en último lugar". Cree que el ser humano es feliz si comprende que la tristeza y la angustia, no son de ellos, son algo externo a ellos que deben ser capaz de rechazar, así explica su estado de felicidad permanente que tanto sorprende (Catalina, ML., Bote, B., García, F. \& Ríos, B., 2005). 


\section{DIFERENCIAS Y SINGULARIDAD DE LA ORTOREXIA}

La ON coincide con los otros TCA, tales como la anorexia y bulimia nerviosas en cuanto que en general existe un deseo de verse perfectos, pero la singularidad de la ortorexia radica en que mientras en aquellas, la obsesión gira en torno a la cantidad de comida consumida, en la ortorexia el eje es la calidad de los alimentos. Los tres trastornos coinciden en castigarse cuando incumplen el plan dietético, pero unos lo harán con ayunos rígidos, otros, con vómitos, purgas, o ambos. Tanto las personas ortoréxicas como las anoréxicas, suelen ser estrictas, rígidas, controladoras, exigentes consigo mismas y con las demás, intentando siempre superarse a si mismo queriendo tenerlo todo bajo control. Suelen ser disciplinadas, tienen deseos de superación y vigilan lo que comen, cuánto y cuándo lo comen.

La pérdida de la sociabilidad es uno de los factores comunes en los TCA, a todas comer les angustia, unas por el hecho de consumir alimentos y otras por el tipo de alimentos, provocando un retraimiento social, un abandono de las amistades y de las actividades de ocio y de recreo, los restaurantes se convierten en lugares prohibidos para sus dietas.

La ON aún no está clasificada en la cuarta edición del Manual Diagnóstico y Estadístico de los Trastornos Mentales (DSM-IV, 1998) ni en la décima edición de la Clasificación Internacional de Enfermedades Mentales (CIE-10,1992), motivo por el que Steven Bratman propone un cuestionario de once preguntas con respuestas afirmativa o negativa, realizado por un profesional o autocumplimentado. Si una persona contesta afirmativamente al menos a cuatro preguntas del cuestionario se podría decir que tiene un problema con la alimentación y permitirá diagnosticar la ortorexia nerviosa (Bratman \& Knight, 2001), (Tabla 1. Auto-Test de Steven Bratman).

\section{FACTORES PSICOSOCIALES, CULTURA- LES Y RELIGIOSOS}

Para poder entender este caso tan novedoso, fue necesario profundizar en el contexto y en los textos, indagando en el significado y sentido del vegetarianismo, analizando las implicaciones y los compromisos con el hinduismo, con el yoga e investigar las influencias y relaciones existentes entre la paciente y esas variables.

Indagando en las doctrinas de la mayoría de las religiones, la esencia es muy similar -el desarrollo espiritual del hombre y la realización del Ser y de Dios como última meta-, Dios es un principio universal.

- El hinduismo y el budismo abrazan el vegetarianismo como parte del camino que conduce a la verdadera santidad. Es necesario adoptar una cosmovisión que incluya dos leyes cósmicas fundamentales: la ley de la evolución y la ley de la reencarnación que deriva en la decisión de no agredir a seres vivos que se encuentran en una etapa evolutiva muy cercana a la del hombre: los animales [4]. Todo ser va evolucionando, vida tras vida, pasando de especies inferiores como los vegetales a otras más adelantadas, como los diferentes tipos de animales, hasta llegar a la forma humana (Gandhi, MK.,1991; Sannuti, A., 2005).

- Entre los vegetarianos existen diferencias en cuanto a lo que comen, motivo que hace necesario referirse a una clasificación (Tabla 2. Clasificación de los vegetarianos) para así poder distinguirlos. La palabra vegetariano fue acuñada por los fundadores de la Asociación Vegetariana Británica en el año 1842 deriva de la palabra latina vegetus, que significa "completo, sano, fresco". Quienes abrazan el ideal del vegetarianismo argumentan que el significado original de esta palabra implica un equilibrado sentido filosófico y moral de la vida, que va mucho más lejos de una mera dieta de verduras y frutas. La base moral del vegetarianismo procede del hinduismo (Gandhi, MK.,1991) [5].

- El yoga [4] une los principios positivos de todas las religiones: la no-violencia, comprensión, ayuda, perdón, caridad; el yoga es un camino espiritual, el cual está basado en la experiencia propia de cada uno y no en las experiencias y reglas de otros, cuyo significado se cambia y se pierde con el curso del tiempo (Asociación Española de Yoga en la vida cotidiana, 2005).

- El cristianismo establecen el ayuno como práctica religiosa relevante y definitoria identificándose como la pureza de corazón delante de Dios. Ayuno y abstinencia estaban asociados a la salvación, penitencia, culpa, ascetismo y huida de lo material. Ayunar es un signo de santidad, es un modelo, una regla y un canon ha seguir. 


\section{DISCUSIÓN}

La conciencia social fue uno de los elementos que favoreció la evolución del ser humano, pues la socialización del ser humano permitió formar grupos cuya necesidad para comunicarse con sus semejantes le obligó a desarrollar el lenguaje. El homo comprobó que para defenderse de los animales y de las inclemencias del tiempo, obtenía mejores resultados cooperando con todos los miembros del grupo que si lo hacía en forma aislada e individual.

Las investigaciones de científicos, entre ellos, antropólogos y paleontólogos, han sido cruciales para conocer mejor nuestro pasado como especie pero también para conocer los aspectos culturales y modos de vida del hombre dejando claro que en una época hace dos millones y medio de años en África evolucionó la primera especie Homo.

“... El caso es que el hombre pudo evolucionar en su forma de alimentación cuando dejó de comer hojas secas y fruta podrida. Se convirtió en carroñero, y aunque pueda parecer un retraso, significó toda una revolución...

“... En un momento de la Historia, un antepasado nuestro decidió roer un hueso y sorber su tuétano. Gracias a ese simple hecho cambió para siempre la historia del hombre, e incluso su fisonomía..." (Arsuaga, JL., 2002)

Estas afirmaciones de Juan Luis Arsuaga, paleontólogo descubridor de la especie Homo Antecesor del yacimiento de Atapuerca en la sierra burgalesa, documenta que la introducción de las proteínas animales en la dieta del Homo permitió su desarrollo como especie, habilitándole para competir con el resto de las especies. Es más, Arsuaga argumenta y defiende que: "la alimentación ha sido esencial para la evolución humana y la clave de esta evolución es la superación del vegetarianismo". El homo descubre que puede cazar carne con instrumentos, convirtiéndose ésta en su alimento preferido y base fundamental de su dieta gracias al descubrimiento fundamental de la producción de lascas de piedras, convirtiéndolas en herramientas eficaces para conseguir alimentos de origen animal (Arsuaga, JL \& Martínez, I., 2000).

...De repente, los humanos tenían acceso a alimentos que previamente les habían estado vedados pues la fabricación de herramientas de piedra habría sido un elemento fundamental de las habili- dades de un comedor de carne; los consumidores de plantas se podían apañar sin artilugios". (Leakey, R., 2000)[7].

Otro de los aspectos, fue el descubrimiento del fuego que marcó el comienzo de otra etapa en la evolución del hombre. No sólo trajo aparejada la tecnología y la posibilidad de calentarse y por tanto de protegerse, sino que le permitió asar, cocer y calentar los alimentos, para una mejor asimilación de éstos por el organismo, provocando con ello que la alimentación fuera esencial para la evolución humana. El cazador tosco dio paso a un hombre más refinado, que empezó a distinguir sabores, colores y aromas, elaborando reglas precisas alrededor de la alimentación, transformando la necesidad de comer en un arte, eligiendo sus ingredientes y creando sus comidas más allá del primitivo acto de supervivencia. Incomprensiblemente, nos encontramos con que los pacientes con ortorexia, cuyas creencias y elecciones alimentarias rechazan precisamente la ingesta de proteínas animales, que fueron fundamentales para la evolución del hombre, arriesgan y comprometen sus vidas por su ideología y convicciones, es más, complicando el proceso de asimilación de los nutrientes eliminando la cocción de los alimentos. Es chocante pensar que tantos años de tradición gregaria, de sociabilidad, de aprendizaje, de solidaridad y de relaciones en la comunidad pueden darse al traste en tan sólo una vida, en este caso en unos años, y acabar aislando, apartando e incomunicando al ser humano.

Richard Leakey (LEAKEY, R., 2000) consideraba que para "el homo" lo impredecible no era el entorno, sino "el otro" y vivir en grupo ocasionaba una alta densidad de relaciones sociales generándose la cultura: "Pero los humanos se hacen humanos por medio de un intenso aprendizaje no sólo de las habilidades de supervivencia sino de costumbres y las convenciones sociales, las leyes de parentesco y las leyes de la sociedad; es decir, de la cultura...; Puede decirse que la cultura es la adaptación humana y es posible gracias al inusual modelo de infancia y maduración".

Precisamente la comensalidad como elemento principal generador y transmisor de cultura al compartir el alimento en grupo y fomentar los vínculos entre los miembros del grupo es lo que actualmente los pacientes con este trastorno de la conducta alimentaria han abandonado, rechazan enérgica- 
mente, encaminándose hacia el aislamiento y la soledad.

Con el paso del tiempo la humanidad se ha instruido, se ha hecho más sabia, se ha enriquecido y han mejoran, en general, las formas y calidades de vida y sin embargo, por paradójico que parezca, cada día es más religiosa. En el mundo, diariamente hay más personas que han roto con sus creencias, abrazando nuevos credos, practicando nuevos ritos, asignando en muchas ocasiones poderes mágicos y cósmicos a objetos, amuletos o personas, aceptando discursos como verdades absolutas que conducen a un sectarismo que busca el aislamiento y la alienación del individuo. Es el fervor religioso que tiene una fuerza única, superior a la política y la economía, a la familia y a la cultura, capaz de vender utopías o fundamentalismos extremos, tanto en su forma de violencia como de no violencia.

Es sorprendente comprobar las innumerables publicaciones sobre vegetarianismo tanto en formato papel como electrónico que existe en el mercado. En muchos portales de la red Internet, informan a los visitantes, cómo iniciarse, dónde dirigirse, dónde realizar las compras de los productos naturales destinados a la alimentación, sobre recetas de cocinas, etc., publicitando y realzando personajes importante tanto del pasado como del presente, que abogaron y eligieron esta opción dietética fundamentando una base moral y ética para practicarla. Para ello, recomiendan al neófito iniciarse con lecturas de carácter científico, religioso, medicinal, filosófico, nutricional o culinario, de fácil comprensión así como aconsejan realizar cursos de cocina vegetariana o algún estudio relacionado. $\mathrm{Al}$ lector, que sólo busca información o documentación, como me ocurrió a mí, me sugirió una forma de sectarismo encubierto en algunos casos, aunque desde las propias páginas se encargaran de decir que no eran sectas ni religión alguna.

He creído interesante recopilar algunos de los pensamientos sobre el ayuno y la ética que defendía y profesaba Mohandas K. Gandhi (Lapierre, D. \& Collins, L., 1983; Ganghi, MK., 1995)) que me han ayudado a entender el comportamiento y estilo de vida de la paciente, que no comparto personalmente, pero que se sustentan en esta filosofía o religión y que demuestran la relación e influencia entre religión-vegetarianismo y ortorexia nerviosa:
"La oración es necesaria para el alma como el alimento lo es para el cuerpo"; "Un alma sana debe habitar un cuerpo sano"; "No considero la alimentación cárnica necesaria para nosotros a ninguna altura de la vida ni bajo cualquier clima en que ordinariamente les sea posible vivir a los seres humanos"; "Nada hay tan poderoso como el ayuno y la oración: nos confieren la necesaria disciplina, espíritu de sacrificio, humildad y firmeza de voluntad, sin lo cual no puede haber progreso real"; "La no violencia es la ley del género humano. La humanidad no puede liberarse de la violencia más que por medio de la no violencia".

\section{CONCLUSIONES}

La visión antropológica ha sido pertinente $\mathrm{y}$ fundamental en este trabajo para comprender e interpretar la fenomenología de este caso clínico, en el que los factores socioculturales fueron determinantes. Muchas de las pacientes, tengan anorexia, bulimia o ortorexia, han convertido su vida en una religión, en un estilo de vida, es según ellas, una opción, equivocada pero una elección. Para unas, la vida se reduce a la báscula, al peso, para otras es la dieta. No tienen conciencia de enfermedad, ni de las consecuencias ni de la gravedad de la situación relacionada directamente con la desnutrición y el bajo peso, negando la repercusión de su conducta alimentaria hasta extremos en que la vida, su vida, corre serios peligros. La clave radica en la actitud hacia la comida, que se considera patológica si cambia el estilo de vida. El ortoréxico se convierte en víctima de su propia dieta. La obsesión gira en torno a la calidad de los alimentos y no en la cantidad de los mismos.

El consumismo de los países ricos, la explosión demográfica, la hambruna, las enfermedades, la sequía y las catástrofes naturales en los países pobres son una combinación perfecta para el desastre ecológico de la humanidad. El mundo está sumido en una crisis medioambiental y la ecología se ha convertido en la religión de ricos.

La mayoría de los expertos coincide en señalar que hay en la sociedad una "tendencia flotante" hacia la ortorexia, que se ha incrementado en los últimos años con la repercusión mediática producida por las crisis alimentaria de las vacas locas [2]. En las sociedades desarrolladas, en las que el problema no es la falta de alimentos sino el exceso, 
cada vez inciden más los TCA. La ortorexia se presenta en niveles socioeconómicos altos y medioaltos y parece no afectar a los sectores marginales ya que este tipo de comida es mucho más cara que la normal y más difícil de conseguir [6]. No cabe duda de que las razones por las que muchas personas adoptan una dieta vegetariana pueden ser muy diversas, de índole religioso, filosófico, motivos de salud, por amor a los animales, por razones humanitarias, o simplemente por estar más cerca de a la naturaleza.

Subrayar y diferenciar que los vegetarianos no son ortoréxicos, que solo las conductas obsesivas y extremas acompañados de sentimientos de soledad, de aislamiento, de creencias e ideología pueden desencadenar esta enfermedad emergente del siglo XXI porque en el fondo lo que subyace son la soledad y el miedo. Al igual que la anorexia y la bulimia, la ortorexia es una forma inadecuada de manejar la angustia.

Al estudiar una enfermedad hay que tener en cuenta que está imbuida de factores culturales que orientan las vivencias del paciente y el enfermero según el Paradigma Interpretativo ha de aprehender la realidad del paciente. Desde la perspectiva enfermera, la interpretación de las situaciones de salud-enfermedad ha facilitado la comprensión, el significado y el sentido de todo lo que acontece convirtiéndose la enfermera, según la definición del profesor Siles, en un agente de comunicación comprensiva e interactiva que intérpreta la realidad del paciente. (Siles, J.,2001).

La paciente de este caso, necesitaba un nuevo planteamiento de vida, un esfuerzo conjunto y multidisciplinar para desconstruir lo construido, como diría Edgar Morin, para desaprender lo aprendido e iniciar un reaprendizaje, pero no siempre se poseen los medios para ello, o las fuerzas, la voluntad o los conocimientos para hacerlo, como argumentaba Virginia Henderson.

\section{NOTAS}

[1] Steven Bratman llevó al extremo su dieta para alcanzar un estado de salud desarrollando esa obsesión patológica por comer sano.

[2] En http://www.nutrar.com/detalle.asp?ID=2222\&opin=1

[3] Síntomas de inanición: astenia, anorexia, disfunicoens hormonales, alteraciones cardiovasculares y electrocardiográficas severas (bradicardia sinussal y alteraciones del ritmo) con posibilidad de muerte súbita.
[4] Doctrina o Principio de Ahimsa: la no violencia basada en el carácter sagrado de la vida.

[5] Vegetarianismo: fundamentos, principios y generalidades en http://www.uva.org.ar/veg.hmtl consultado el 23/02/05

[6] Durante los 48 días que duró su ingreso sólo en Tofú (queso de soja) he calculado el costo del mismo que se elevó a $409.37 €$ ( 150 gr. /día X $48=7200$ gr.; un paquete de 350 gr. de de tofú cuesta $19.9 €=409.37 €$ ) a los que hay que añadir el valor de los kilos de fruta fresca, de los frutos secos, del pan de cereales, etc.) elevando el gasto alimenticio a un valor que sin dudas las familias con rentas bajas les sería imposible mantener.

\section{BIBLIOGRAFÍA}

- ARSUAGA, JL \& MARTÍNEZ, I. (2000) "La especia elegida. La larga marcha de la evolución humana”. Ed. DeBolsillo. Temas de Hoy. Barcelona.

- ARSUAGA, JL. (2002) "Los Aborígenes, la alimentación en la evolución humana”. Ed. RBA Libros, S.A., Barcelona.

- ASOCIACIÓN ESPAÑOLA DE YOGA EN LA VIDA COTIDIANA, "Vegetarianismo" en salud y vida natural, consultado en http://yoga-en-la-vida-cotidiana.org/gegetarianismo.htm el 26 de enero de 2005.

- BRATMAN, S. \& KNIGHT, D. (2001) "Health food junkies. Ortorexia nervosa: overcoming the obsesión with healthful eating. Brodway Boooks, New York.

- CATALINA, ML., BOTE, B., GARCIA, F. \& RIOS, B., 2005:

"Ortorexia nerviosa. ¿Un nuevo trastorno de la conducta alimentaria". Actas Española de Psiquiatría; 33(1):66-68, Madrid

- CIE-10, (1992) "Clasificación Internacional de enfermedades”. Organización mundial de la salud, Madrid.

- DSM-IV, (1998) "Manual diagnóstico y estadístico de los trastornos mentales", Ed. Masson, Barcelona.

- FERNÁNDEZ, F y TURÓN, V. (1998) "Trastornos de la alimentación. Guía básica de tratamiento en anorexia y bulimia"; Ed. Masson S.A., Barcelona.

- GANDHI, MK. (1991) "La base moral del vegetarianismo", en Historia del vegetarianismo: Mohandas Gandhi, en Unión Vegetariana Internacional (IVU) consultado el 14/02/2005 en http://www.ivu.org/spanish/news/ivu/other/gandhi2.html.

- LAPIERRE, D. \& COLLINS, L., (1983) "Esta noche la libertad. Triunfo y tragedia de Gandhi"; Ed. Plaza \& Janet S.A., Circulo de lectores, Barcelona.

- LEAKEY, R., (2000) "El origen de la humanidad", Ed. Debate S.A. Barcelona.

- SANNUTI, A., Historia del vegetarianismo: Mohandas Karamchand Gandhi, en Unión Vegetariana Internacional (IVU) consultado en http://www.ivu.org/spanish/history/ gandhi el 14 de febrero de 2005

- SILES GONZÁLEZ J. (2001) Los textos como fuentes de conocimientos en Ciencias Sociales y Enfermería. Una aplicación de la metodología cualitativa. Index de enfermería (versión digital); 32-33. Disponible en http://www.indexf.com/index-enfermeria/32-33revista/32-33_articulo_2834.php consultado el 6 de mayo de 2006.

- Vegetarianismo: Fundamentos, principios y generalidades en http://www.uva.org.ar/veg.hmtl consultado el 23/02/05. 


\begin{tabular}{|l|}
\multicolumn{2}{|c|}{$\begin{array}{c}\text { Tabla 1. Auto-Test de Steven Bratman } \\
\text { (Bratman \& Knight, 2001) }\end{array}$} \\
\hline 1. ¿Pasa más de tres horas al dia pensando en una dieta sana? \\
\hline 2. ¿Se preocupa más de la calidad de los alimentos que del placer de \\
comerlos? \\
\hline 3. ¿Le ha ocurrido que conforme aumenta la calidad de su comida \\
disminuye la calidad de su vida? \\
\hline 4. ¿Se siente culpable cuando se sa Ita alguna de sus convicciones \\
dietéticas? \\
\hline 5. ¿Planifica hoy lo que va a comer mañana? \\
\hline 6. ¿Le aísla socialmente su manera de comer? \\
\hline 7. ¿Gasta mucho dinero en productos alimenticios sanos? \\
\hline 8. ¿Se ha vuclto más cstricto(a) consigo mismo(a)? \\
\hline 9. ¿Aumenta su autoestima cuando come saludable? \\
\hline 10. ¿Infravalora a los que no comen saludablemente? \\
\hline 11. ¿Evita comer alimentos con los que antes disfrutaba? \\
\hline
\end{tabular}

Tabla 2. Clasificación de los vegetarianos

- VEGETARIANO no incluye carnes de ningún tipo.

- OVO-LACTO-VEGETARIANO: incluye derivados de animal, como huevosy lácteos.

- OVO-VEGETARIANO: incluye solo huevos.

- LACTO-VEGETARIANO: incluye lácteos de origen animal y sus derivados.

- API designa a quien consume miel

- VEGETARIANO VEGANO: dieta exclusivamente basada en alimentos de origen vegetal, sin excepciones de ningün tipo, excluyendo también en todo el ámbito de su vida el uso de derivados de animales o de productos que hayan implicado para su fabricación o producción la explotación y/o el sufrimiento de algún animal. No utiliza en su vida, los derivados de la matanza y/o explotación de animales, como cuero, lana, seda, cosméticos, etc, oponiéndose también a todo experimento. El vegano aborrece las prácticas inherentes a la cria de ganado y productos de granja, pero mucha gente también lo hace por razones de salud, ecológicas, espirituales, etc.

- CRUDIVORO VEGETARIANO: El crudivoro no cocina ni calienta las comidas, ingiere todo en $\$ \mathrm{u}$ estado natural: frutas, verduras, nueces, semillas, legumbres, cereales, brotes, etc :

- FRUGIVORO, FRUTISTA OF RUTARIANO: se alimenta exclusivamente de frutos, generalmente crudos y de época. 